\title{
Potere e territorio. Gli Achemenidi nei GEOGRAPHik̇̀ DI STRABONE
}

\author{
Omar Coloru
}

ArScAn HAROC (Nanterre)

\begin{abstract}
Although posterity knew Strabo only as the "Geographer," he nonetheless conceived the Geographikà as a complement to his Historikà hypomnemata, a continuation of the Histories of Polybius. Throughout his geographical work, it is possible to find many references concerning the history of the Greek world, and not only. In this framework, the Achaemenid Empire is present in the form of anecdotes on historical, ethnographic as well as linguistic aspects of the Persian world. Particular attention is devoted to those kings who played a prominent role in the confrontation between the Greeks and the barbarians (i.e. Cyrus II, Darius I, Xerxes and Darius III), while the other monarchs are nothing more than shadows. From the analysis of the passages on the Achaemenids, it is possible to argue that, in Strabo's eyes, the Persians did not have an exclusively negative image when compared to the Parthians, and special emphasis is placed on their capability in shaping and modifying the geographical space through their technical knowledge.
\end{abstract}

Key words: Achaemenid Empire, Persia, Strabo, landmark, ancient geography.

Negli ultimi decenni lo studio della percezione dei barbari nei Geographikà di Strabone ha ricevuto un'attenzione sempre crescente da parte degli studiosi. In effetti, a partire dal lavoro di Thollard ${ }^{1}$ pubblicato nel 1987 si sono aggiunti nel corso degli anni altri importanti contributi come quelli di Drijvers e Dąbrowa sui Parti in Strabone ${ }^{2}$ (ma si veda anche in ambito più generale il lavoro di Lerouge-Cohen ${ }^{3}$ ), mentre fra i più recenti saggi sull'immagine del barbaro nell'opera dell'autore di Amasea possiamo citare il lavoro pubblicato da Almagor nel 2005. ${ }^{4}$ Inoltre, nel 2011 è stata data alle stampe, sotto la direzione di Dominique Lenfant, la collezione di tutti i riferimenti all'impero achemenide e alla civiltà persiana che è possibile reperire nelle opere degli autori greco-romani: è nell'ambito di questo lavoro che Stéphane Lebreton ha curato la sezione dedicata

\footnotetext{
1 Thollard 1987.

2 Drijvers 1998, 279-293; Dąbrowa 2015, 285-304.

${ }^{3}$ Lerouge-Cohen 2007.

${ }^{4}$ Almagor 2005, 42-55.
} 
a Strabone. ${ }^{5}$ Questo contributo permette senza dubbio al lettore di avere una visione di insieme chiara e di rapida consultazione sull'immagine dei Persiani nell'opera straboniana. È proprio utilizzando come punto d'appoggio questa raccolta di testimonianze che vorrei proporre alcune considerazioni in merito all'impero achemenide così come viene presentato nei Geographikà.

\section{La dinastia achemenide nei Geographikà}

In primo luogo è opportuno cominciare la nostra analisi passando in rassegna i sovrani achemenidi menzionati nel corso della trattazione di Strabone, in modo da comprendere in quale contesto e perché essi vengano chiamati in causa. Per ogni sovrano verrà presentata una piccola tabella riassuntiva che illustra sinteticamente il contenuto delle citazioni.

\section{Ciro II}

\begin{tabular}{|l|l|}
\hline XI.6.2 & Spedizione di Ciro contro i Massageti \\
\hline XI.8.6 & Ciro sconfigge i Saka e istituisce le feste Sacee \\
\hline XI.13.5 & Ciro toglie all'Armenia il potere sull'Asia \\
\hline XI.13.5 & Ciro sconfigge Astiage \\
\hline XIII.4.13 & Il toponimo Kyrou pedion deve il suo nome ai Persiani \\
\hline XV.1.25 e XV.2.5 & Spedizione fallita di Ciro contro gli Indiani \\
\hline XV.2.10 & Ciro e gli Evergeti \\
\hline XV.3.2 & Ciro trasferisce la capitale a Susa \\
\hline XV.3.6 & $\begin{array}{l}\text { Ciro avrebbe preso il suo nome dal fiume omonimo presso Pasargade, in } \\
\text { realtà si sarebbe chiamato Agradates }\end{array}$ \\
\hline XV.23 & Ciro diventa re dei Persiani \\
\hline
\end{tabular}

Il fondatore della potenza persiana è ricordato in modo particolare per la sua dimensione eroica e quasi mitologica. Oltre alla vittoria sul re di Media Astiage, Strabone fa riferimento alle campagne in Armenia e soprattutto a quelle contro gli Sciti e i Massageti e alle spedizioni orientali in Drangiana e in Gedrosia. Quest'ultima in particolare è degna di nota in quanto il geografo riporta la notizia che Ciro era associato alla figura della mitica Semiramide come esempio di grandi condottieri che non sono riusciti a sottomettere l'India a differenza di Alessandro. Interessante anche la leggenda in base alla quale il fondatore del regno avrebbe preso il suo nome dall'omonimo fiume che scorre presso Pasargade, mentre si sarebbe in realtà chiamato Agradate (probabile errore da parte di Strabone nella trasmissione della notizia fornita da Ctesia di Cnido che parla invece di un Atradate). ${ }^{6}$ È forse questa l'eco di una delle leggende che circolavano sul fondatore

\footnotetext{
5 Lenfant 2011; Lebreton 2011, 374-389.

${ }^{6}$ Ctesia di Cnido F 8d (ed. Lenfant 2004).
} 
dell'impero? Il fatto che il sovrano assuma il nome di un fiume fa pensare in effetti alla figura dell'eroe reale che riesce a dominare gli elementi. D'altra parte, un parallelo interessante sul controllo dei fiumi da parte dei sovrani persiani è la leggenda riportata da Erodoto (III.117) a proposito di un Gran Re che avrebbe chiuso le cinque braccia del fiume Akes con altrettante dighe trasformando la piana in cui scorreva in un grande lago. L'impresa si sarebbe svolta nell'Iran settentrionale in un'area in cui si approvvigionavano i Corasmi, gli Ircani, i Parti, i Sarangi e i Tamanei. Il fatto che l'identità di questo Gran Re non sia specificata lascia supporre che la figura di questo sovrano nasconda da un lato una figura eroica mitologica che ha il potere di dominare le forze della natura, mentre dall'altro serva da racconto eziologico per spiegare il controllo esercitato dagli Achemenidi sulle risorse idriche. ${ }^{7}$

\section{Cambise}

\begin{tabular}{|l|l|}
\hline XV.3.24 & Cambise succede a Ciro \\
\hline XVII.1.5 & $\begin{array}{l}\text { Cambise conquista l'Egitto; Meroe chiamata così in onore della sorella di } \\
\text { Cambise }\end{array}$ \\
\hline XVII.1.27, 46,54 & Follia di Cambise; armata perduta di Cambise \\
\hline
\end{tabular}

Pochi sono i riferimenti al figlio e successore di Ciro, praticamente limitati alla trattazione sull'Egitto. In effetti qui Strabone sembra basarsi solo sulla narrazione erodotea a proposito della follia di Cambise e degli atti di empietà da lui commessi contro la religione egiziana. Non manca l'episodio dell'armata perduta nel deserto libico, ma si fa anche riferimento all'etimologia del toponimo Meroe, che deriverebbe dal nome della sorella di Cambise. ${ }^{8}$

\section{Dario I}

\begin{tabular}{|l|l|}
\hline VII.3.8, 9,15 & Spedizione di Dario contro gli Sciti; costruzione di un ponte di barche sull'Istro \\
\hline VII.3.14 & Dario bloccato nel deserto dei Geti dopo aver passato l'Istro \\
\hline XIII.1.22 & $\begin{array}{l}\text { Dario fa incendiare Abido e le poleis della Propontide dopo la campagna contro } \\
\text { gli Sciti }\end{array}$ \\
\hline XIV.1.7 & $\begin{array}{l}\text { Il poeta tragico Frinico multato dagli Ateniesi per aver scritto la Presa di Mileto } \\
\text { da parte di Dario }\end{array}$ \\
\hline XIV.1.17 & Dario nomina Syloson tiranno di Samo \\
\hline XV.3.24 & Dario succede al falso Mago \\
\hline XVI.1.3 & $\begin{array}{l}\text { Il villaggio di Gaugamela aveva il compito di custodire e nutrire il cammello che } \\
\text { aveva riportato sano e salvo Dario dalla spedizione contro gli Sciti }\end{array}$ \\
\hline
\end{tabular}

${ }^{7}$ Cfr. Briant 2002, 415-416. La storia attinge a un patrimonio di leggende indo-arie sulla regalità e che hanno al centro l'elemento acquatico, si veda ancora Briant 2002, 239-240.

${ }^{8}$ Cfr. Diodoro Siculo, I.33. 
Più visibilità ha senza dubbio la figura di Dario I di cui si mette in risalto l'attività militare, e in particolare le due spedizioni che lo avevano reso celebre: la campagna contro gli Sciti e quella in Grecia nel corso della Prima guerra persiana. Vengono poi riferiti alcuni aneddoti di origine erodotea legati alla nomina di Syloson a tiranno di Samo, ma oltre a ciò è anche presente un breve accenno agli eventi che portarono alla sua ascesa al trono dopo aver ucciso il falso mago Gaumata.

\section{Serse}

\begin{tabular}{|l|l|}
\hline I.1.17 & Spedizione di Serse in Grecia \\
\hline VII, fr. 33 e 35 & Scavo del canale del Monte Athos \\
\hline XIII.1.12 & Serse "dona" Lampsaco a Temistocle \\
\hline XIII.1.22 & Serse fa costruire il ponte di barche sull'Ellesponto \\
\hline XI.11; XIV.1.5; XVII.1.43 & Serse e i Branchidi \\
\hline XIV.1.10 & Serse "dona" Miunte, Lampsaco e Magnesia a Temistocle \\
\hline
\end{tabular}

I passi dedicati a Serse, come è lecito aspettarsi, sono quelli legati alla Seconda guerra persiana. Inoltre Strabone ricorda più volte la storia del tradimento dei Branchidi e del loro esilio in Battriana. In almeno due passi, poi, si fa riferimento alle vicende di Temistocle che per il suo mantenimento ricevette da Serse i proventi delle città di Miunte, Lampsaco e Magnesia.

\section{Artaserse I}

\begin{tabular}{|l|l|}
\hline I.3 & Siccità durante il suo regno \\
\hline XV.3.21 & Chiamato Longimano (ma Strabone attribuisce il soprannome a Dario) \\
\hline
\end{tabular}

Solamente due le notizie riportate nel testo di Strabone a proposito di questo sovrano. Nel primo caso, si riporta una notizia di Xanto di Lidia nella citazione di Eratostene in base a cui si registrò un periodo di siccità durante il regno di Artaserse. Il secondo riferimento riguarda il soprannome del sovrano, Macrocheir, seguito dalla spiegazione letterale in base alla quale il sovrano sarebbe stato chiamato così perché avrebbe avuto dei lunghi arti. Tale etimologia è la stessa che si ritrova più tardi in Plutarco, ma è molto probabile che il termine greco non sia altro che la traduzione letterale di una titolatura persiana che fa riferimento alla potenza del sovrano. ${ }^{9}$ Da notare poi che Strabone commette un errore attribuendo questo soprannome a Dario. Questo tipo di errore non sembra essere il primo di questo tipo commesso da Strabone, tant'è che una confusione simile si ritrova, ad esempio, nella narrazione dell'indipendenza della Battriana (XI.9.2), che il geografo attribuisce erroneamente a Eutidemo invece che a Diodoto.

9 Plutarco, Artaserse, 1,1. L'etimologia più probabile è quella legata all'epiteto, frequente nell'epica persiana, di Derāz-dast, dove dast, oltre a significare "mano", indica anche "potere", "autorità". Solo Polluce, Onomasticon II.151 sembra consapevole del fatto che il titolo vada inteso in senso metaforico, vedi von Gall 1990, 110; Tafażżolī 1994, 320. 


\section{Arses - Artaserse IV}

XV.3.24 Successione al trono attraverso le trame dell'eunuco Bagoa

\section{Dario III}

\begin{tabular}{|l|l|}
\hline II.1.24 & Itinerario di Dario III da Gaugamela alle Porte Caspie \\
\hline XII.3.10 & Dario regna all'epoca di Alessandro \\
\hline XVI.1.3 & Sconfitta di Dario presso il villaggio di Gaugamela \\
\hline XVI.3.5 & Dario manda in esilio Mithropastes nell'isola di Tyrine \\
\hline XVI.3.17 & $\begin{array}{l}\text { Dario non è il legittimo successore al trono achemenide. La dinastia si fermerebbe } \\
\text { con Arses }\end{array}$ \\
\hline
\end{tabular}

Pochi sono anche i riferimenti all'ultimo sovrano achemenide, fatto che trasforma Dario in una personalità evanescente, quasi completamente oscurata dalla figura di Alessandro Magno. Di conseguenza Dario III viene evocato a proposito dei luoghi in cui si scontrò coi Macedoni o dell' itinerario che seguì durante la sua fuga nelle Satrapie Superiori. Da notare inoltre che Strabone accetta e riporta la notizia dell'illegittimità di Dario III, una storia frutto della propaganda macedone che era stata utilizzata per rafforzare le pretese imperialiste di Alessandro sull'impero persiano. ${ }^{10}$

Come si può vedere da questa rapida rassegna, fra il regno di Artaserse e quello di Dario III esiste un vero e proprio vuoto che comprende tutti i sovrani che si succedettero al trono in questo intervallo cronologico (Serse II, Sogdiano, Dario II Ochos, Artaserse II, e Artaserse III). Questa situazione non sembra dovuta al caso, ma si configura come una scelta deliberata di Strabone. In effetti, se osserviamo il paragrafo dedicato alla storia dinastica dell'Impero achemenide, ci si accorge senza difficoltà che la lista dei sovrani citati comprende a grandi linee quasi tutti i re che sono effettivamente menzionati nell'insieme dei Geographikà:

Dunque Ciro fu colui che li (sott. i Persiani) rese egemoni. A lui successe il figlio Cambise, che venne rovesciato dai Magi. In seguito essi furono eliminati dai Sette Persiani, che conferirono il potere a Dario, figlio di Istaspe. Poi i suoi successori terminarono con Arses, che fu assassinato dall'eunuco Bagoa, il quale a sua volta mise sul trono un Dario che non era di stirpe regale (XV.3.24).

Lo stesso schema sembra applicarsi anche ai membri della famiglia reale e agli ufficiali achemenidi ricordati da Strabone. Abbiamo infatti i casi seguenti.

\section{Regno di Ciro}

Arpago, generale di Ciro, autore della conquista persiana dell'Asia Minore, ricordato perché all'avvicinarsi del suo esercito gli abitanti di Focea fuggirono verso Kyrnos e Massalia (VI.1.1).

\footnotetext{
${ }^{10}$ Cfr. Briant 2002, 770-772.
} 


\section{Regno di Cambise}

Meroe (XVII.1.5), presunta sorella di Cambise.

\section{Regno di Dario I}

Datis (IX.1.22), generale sconfitto da Miliziade a Maratona.

Oronte (XI.14.15), il fondatore della dinastia degli Orontidi di Armenia che discenderebbe da Idarne, uno dei Sette Persiani che avevano eliminato Gaumata:

Questa, dunque, è la storia antica (dell'Armenia), ma converrebbe riassumere anche quella più recente che va dai Persiani fino ai giorni nostri: i Persiani e i Macedoni si impadronirono dell'Armenia, dopodiché fu la volta di coloro che tenevano la Siria e la Media; per ultimo venne Oronte, il discendente di Idarne, uno dei Sette Persiani.

\section{Regni di Dario e di Serse}

Mardonio (IX.2.31), genero di Dario, generale suo e di suo figlio Serse. Viene ricordato a proposito della sua morte sul campo di battaglia di Platea insieme a 300.000 Persiani.

\section{Regno di Serse}

Megabate (IX.2.9), ammiraglio della flotta di Serse viene ricordato per aver messo a morte Salganeo, l'uomo che avrebbe fatto da guida alla flotta e poi accusato di tradimento.

Da qui in poi si passa direttamente alla fase finale dell'impero sotto Dario III:

Amastri (XII.3.10), figlia di Oxyathres, fratello di Dario III. Strabone riferisce che era la moglie di Dionisio, tiranno di Eraclea Pontica.

Atropate (XI.13.1), satrapo della parte occidentale della Media che da lui prende il nome di Media Atropatene. Riesce a impedire ai Macedoni di conquistare questa regione e in seguito fonda una dinastia personale.

Mazenes (XVI.3.7), governatore dell'isola di Doracta nel Golfo Persico (o Oaracta o Duracta, corrispondente alla moderna Kishm, Iran), divenuto poi pilota della flotta macedone. ${ }^{11}$

Memnone di Rodi (XIII.1.46-70), ufficiale al servizio di Dario III, ricordato per aver arrestato con l'inganno e inviato alla corte del Gran Re il tiranno di Asso, Ermia.

Mithropastes (XVI.3.5), figlio del satrapo di Frigia Arsite, rifugiatosi nell'isola di Tyrine nel Golfo Persico, perché esiliato da Dario III.

Spitamene e Besso (XI.8.7) sono menzionati invece per il fatto di essersi rifugiati presso popolazioni d'Asia Centrale di fronte all'avanzata di Alessandro Magno. Inoltre si fa brevemente riferimento alla loro morte (XI.11.7): assassinio per mano dei barbari nel caso di Spitamene, cattura e poi condanna a morte da parte dei Persiani nel caso di Besso. Sempre a proposito degli scontri che il Macedone dovette sostenere nella regione vengono ricordati Ariamazes, Sisimithres, Oxyarthes e la figlia Rossane, futura sposa di Alessandro (XI.11.4).

\footnotetext{
11 Potts 2010, 523-534.
} 
Al di fuori di questa lista si collocano Omane e Anadate (XII.8.4-5), considerati da Strabone dei generali persiani che avrebbero sconfitto i Saka e istituito le feste Sacee a Zela di Cappadocia, ma che in realtà sono due divinità iraniche venerate insieme ad Anahita e delle quali la prima era associata sincretisticamente a Zeus. ${ }^{12}$

Da quanto si è visto finora, è lecito ritenere che Strabone abbia preferito concentrare la sua attenzione e quella dei suoi lettori sui regni che ebbero un impatto maggiore a livello storico e ideologico nello scontro di civiltà tra mondo greco e mondo dei barbari. In effetti è necessario entrare nell'ottica dell'autore e ricordare le finalità che egli si era posto al momento della redazione dei Geographikà: Strabone non sta scrivendo un'opera storica, che è già stata composta e che per di più non riguarda il periodo che ci interessa qui. Di conseguenza non dobbiamo attenderci notizie approfondite sulla storia achemenide. Strabone, infatti, si muove nel solco della tradizione classica e in genere si basa sul testo di Erodoto per il periodo delle Guerre persiane, e degli storici di Alessandro per quanto riguarda gli ultimi anni dell'Impero achemenide. Questa scelta è dettata dall'agenda di Strabone. L'autore, in effetti, si rivolge ad un pubblico di lettori - quello degli uomini politici romani - che in un certo qual modo si aspetta di vedere illustrato il primato della civiltà greca (e romana) sul Barbaro. Ciononostante, nel corso dell'esposizione emergono aspetti rilevanti che mostrano come i Persiani abbiano lasciato una traccia duratura della loro presenza sul territorio o siano riusciti a creare dei lieux de mémoire. A questo proposito possiamo suddividere i dati presenti nei Geographikà in più sezioni.

\section{Luoghi o edifici legati allo scontro con i Greci}

I passi che descrivono questi luoghi mostrano in generale i Persiani nella veste di barbari accecati dalla superbia che finisce per essere punita con la sconfitta militare. Molti esempi si trovano, come è facile aspettarsi, nei libri dedicati alla Grecia: in Attica è inevitabile citare la battaglia di Salamina (IX.1.9), Nelle vicinanze di Anaphlystos c'è il tempio di Afrodite Colias dove le onde portarono i resti delle navi persiane naufragate a Salamina. (IX.1.21); Capo Sepias (IX.5) celebrato in tragedie ed inni per la distruzione totale della flotta di Serse; segue poi la piana di Maratona (IX.1.17 e 1.22). Nella sezione dedicata alla Beozia Strabone ha modo di ricordare la battaglia di Platea (IX.2.5 e 31).

\section{Persiani in battaglia}

Negli scontri coi Greci, si può notare che in alcuni casi le vittorie dell'esercito achemenide sono presentate in una luce tale da sminuirne la gloria o vengono comunque ridimensionate dal fatto di essere state ottenute attraverso il ricorso a una smodata violenza da parte dei Persiani o in cui paradossalmente sono gli sconfitti a ricoprirsi di gloria. Il caso più eclatante è quello delle Termopili (Locride) che fu il teatro dello scontro leggendario con i 300 spartiati di Leonida. Strabone in questo caso descrive il monumento ai caduti spartani e quelli di Opunte e si mette a ricordare le imprese di Leonida (IX.4.2 e 16). A X.3.8 il geografo accenna all'ammirazione che i Persiani ebbero per gli Spartani

\footnotetext{
${ }^{12}$ Cfr. Saprykin 2009, 259, 263-265, 267.
} 
al momento di ingaggiare battaglia, mentre prima dello scontro li avevano derisi, perché si erano presentati al combattimento con i capelli ben pettinati. D'altra parte, però, sempre a proposito di questo scontro, si dovrà segnalare che i Persiani sono visti in una luce più favorevole nel libro I (1.17), in quanto Strabone ricorda che sfruttando le conoscenze geografiche fornite loro da Efialte essi furono in grado di avere la meglio sugli Spartani che proteggevano il passo delle Termopili.

Nell'ambito dei successi militari persiani, la figura di Ciro ancora una volta sembra godere di un trattamento migliore rispetto ai suoi successori. Diversi passi in effetti celebrano le sue vittorie sui Massageti e sugli Sciti (XI.8.4-6).

Il tema della violenza persiana si ritrova nel riferimento alla distruzione di Eretria e alla deportazione dei suoi abitanti (X.1.10 e XVI), all'incendio di Abido e delle altre città della Propontide da parte di Dario I (XIII.1.22), alla distruzione del tempio di Didima e dei santuari della Ionia da parte di Serse (XIV.1.5), alla distruzione della tomba di Belos a Babilonia sempre per volontà di Serse o ancora alle tracce delle distruzioni inflitte da Cambise ai templi e agli obelischi egiziani (XVII.1.27 e 46).

\section{Persiani costruttori}

Un carattere particolare che emerge dall'esposizione di Strabone è la perizia tecnica dimostrata dai Persiani sia che essa venga giudicata secondo il cliché della hybris sia secondo quello dell'ostentazione del lusso. ${ }^{13}$ Come abbiamo anticipato, l'intervento persiano lascia in ogni caso un segno sul territorio sia nella sua qualità di luogo della memoria nello spazio geoculturale greco sia come punto di riferimento geografico. Questa fusione tra dato storico e spazio geografico non è semplicemente dettata da una volontà di abbellimento dell'esposizione o da sfoggio di erudizione, ma risponde anche ad un'esigenza pratica di creare una mappa mentale del mondo attraverso la creazione di precisi punti di riferimento, di veri e propri landmark.

In Beozia l'ammiraglio Megabate fa erigere un monumento funebre per Salganeo, la guida da lui sospettata ingiustamente di tradimento e fatta giustiziare (I.1.17 e IX.2.9). In Lidia troviamo invece un'imponente piattaforma di osservazione fatta costruire dai Persiani sulla cima del monte Tmolo allo scopo di controllare la piana del fiume Caistro (XIII.4.5). Nella sezione sulla Persia Strabone dedica un certo spazio alle descrizioni della tomba di Ciro riportate dagli storici di Alessandro, mentre al contrario si limita a un brevissimo riferimento all'iscrizione funebre che si sarebbe trovata sulla tomba di Dario (XV.3.7-8).

In questo ambito possiamo far rientrare anche i passi in cui si accenna alle residenze reali persiane di Susa (XV.3.2), Pasargade, Persepoli, Gabai (presso la moderna Isfahan) e Taoke sul Golfo Persico (XV.3.2-3 e 6). Inoltre, tra Gaugamela e Babilonia si trova la località di Sadrakai, dove sorgeva la residenza preferita di Dario I (XVI.1.4).

L'attività di controllo del territorio si realizza anche nella fondazione di insediamenti, come la colonia di Hyrcanis in Lidia dove i re achemenidi avevano insediato dei soldati provenienti dall'Ircania. La città ha poi dato il suo nome anche alla piana omonima (XIII.4.13). ${ }^{14}$ Nella stessa area Ciro lascia traccia del suo passaggio nella piana chiamata

\footnotetext{
13 Rollinger 2013, 73.

14 Sulla colonia di Hyrcanis si veda Cohen 1995, 209-212.
} 
Kyrou pedion (ibid.), ma anche a Cyreschata, la città più orientale dell'impero persiano, fondata sulla riva dello Iassarte (Syr-darya).$^{15}$ Vengono poi le rocche di Ariamaze e Siismitre e la "città dei Branchidi" dove Serse aveva insediato i sacerdoti del tempio di Didima (XI.11.4). ${ }^{16}$

\section{I signori delle acque}

Abbiamo già accennato in precedenza ai miti legati alla figura del sovrano achemenide come dominatore degli elementi e in particolare delle acque. Su questo particolare aspetto Strabone fornisce a più riprese degli esempi legati per lo più ad episodi di storia militare. In tale contesto, il nostro autore menziona il ponte di barche costruito da Dario sul Bosforo e sull'Istro (Danubio) al momento della sua campagna contro gli Sciti: ${ }^{17}$

\begin{tabular}{|l|l|}
\hline VII.3.9 & $\begin{array}{l}\text { Citazione di un poema di Cherilo di Samo intitolato L'attraversamento sul ponte di } \\
\text { barche dedicato all'opera di ingegneria militare compiuta sull'Istro per volontà di } \\
\text { Dario. }{ }^{17}\end{array}$ \\
\hline VII.3.15 & $\begin{array}{l}\text { Localizzazione del ponte di barche di Dario nella parte inferiore dell'isola di Peuce } \\
\text { (=Isola dei pini) nel delta dell'Istro. }\end{array}$ \\
\hline VII.3.14-15 & L'esercito persiano attraversa l'Istro. \\
\hline
\end{tabular}

Diversi sono anche i riferimenti al ponte di barche fatto costruire dai genieri persiani per ordine di Serse in modo da collegare l'Asia all'Europa e permettere all'esercito di attraversare l'Ellesponto. A questo proposito Strabone identifica il punto di inizio della costruzione nel Capo Sestias presso Abido (fr. 55 del libro VII e XIII.1.22). ${ }^{18}$

$\mathrm{Ci}$ sono poi opere di ingegneria idraulica compiute o almeno progettate dai sovrani achemenidi. Dario I, ad esempio, viene ricordato per aver continuato i lavori di scavo del canale tra Bubastis e il Mar Rosso, opera che avrebbe però lasciato incompiuta per timore che le acque del mare sommergessero l'Egitto (XVII.1.25). Quanto a Serse, Strabone parla dello scavo del canale del Monte Athos (fr. 33 e 35 del libro VII). Inoltre, pare che lo stesso re avesse progettato di riempire il braccio di mare tra il capo Amfiale e l'isola di Salamina con una diga, ma che avesse dovuto accantonare l'idea a causa della sconfitta subita (IX.1.13).

Due passi rivelano l'attenzione portata dai sovrani achemenidi allo sfruttamento dei corsi d'acqua. In primo luogo Strabone riferisce che l'ambasciatore ateniese Diotimo figlio di Strombico ${ }^{19}$ aveva descritto il suo itinerario per raggiungere Susa (437-436 a.C.) allo storico Damaste e che questo itinerario era stato a sua volta riportato da Eratostene

15 Anche in questo caso Cyreschata, "Kura, la più lontana" è probabilmente un'etimologia popolare del toponimo iranico Kuruš-ka9a, "Il forte/città di Ciro", cfr. P'yankov 1993, 514.

${ }_{16}$ Sull'identificazione e localizzazione dei siti di queste due fortezze, cfr. Rapin 2013, 43-82.

${ }^{17}$ Per un'analisi recente del poema di Cherilo si veda Cucinotta 2011, 97-118.

${ }_{18}$ Sul ponte di Serse e le interpretazioni dei rituali compiuti dal re per propiziare la traversata dell'Ellesponto: Dan 2015, 191-235.

${ }^{19}$ L'epigrafia ateniese ha permesso di accertare l'esistenza e la carriera di questo personaggio, cfr. Develin 2003, 99, 104, 107, 458. 
(I.3.1). L'itinerario, della durata di 40 giorni, avrebbe previsto la navigazione da Atene fino alle coste della Cilicia, da dove l'ambasciatore avrebbe proseguito lungo il fiume Cidno per poi passare all'Eufrate, al Tigri e al Choaspe fino a Susa. In polemica con Eratostene, Strabone critica aspramente l'affidabilità di queste informazione, tuttavia Pierre Briant ha mostrato che questo itinerario ha buone probabilità di essere attendibile nelle sue grandi linee se ipotizziamo che il tragitto dal Cidno all'Eufrate sia stato fatto per via di terra. Imbarcandosi sull'Eufrate a Tapsaco, l'ambasceria guidata da Diotimo avrebbe raggiunto la Babilonia meridionale, dove la fitta rete di canali permetteva effettivamente di raggiungere Susa per la via d'acqua. ${ }^{20}$

Il secondo esempio è quello delle cataratte del Tigri e dell'Eufrate che secondo gli storici di Alessandro sarebbero state delle barriere per impedire alle imbarcazioni nemiche di assalire la regione dall'esterno. Il Macedone di conseguenza le avrebbe fatte distruggere (XVI.1.9). In realtà queste costruzioni erano delle dighe da utilizzare a scopo di irrigazione. ${ }^{21}$

Il tema delle acque viene ricordato anche a proposito dell'etimologia del Mar Eritreo (Golfo Persico) in due passi dei Geographikà, nello specifico XVI.3.5 e 4.20. Strabone sostiene che il mare tragga il suo nome da un leggendario sovrano chiamato Erythras. I Persiani avrebbero trasmesso questa storia ai Greci e Strabone ne riporta due versioni. La fonte della prima versione è il persiano Mithropastes che sull'isola di Tyrine mostrò a Nearco la tomba del sovrano che aveva regnato su quella regione e aveva donato il suo nome al mare. La sepoltura consisteva in un grande tumulo su cui crescevano delle palme. ${ }^{22}$ La seconda versione, più dettagliata, sarebbe stata trasmessa da un uomo di origine persiana residente ad Atene di nome Boxos e poi riportata dallo storico Agatarchide di Cnido nella sua monografia sul Mar Rosso. ${ }^{23}$ Anche in questo caso si fa riferimento a Erythras senza che però vengano forniti ulteriori informazioni sul suo conto se non che si trattava di un persiano. Il racconto tramanda che questo personaggio era partito alla ricerca di una mandria di cavalli messa in fuga da una leonessa. Gli animali si erano dunque spinti fino alla costa e da lì avevano raggiunto l'isola a nuoto. Per raggiungere i cavalli Erythras avrebbe costruito una zattera diventando così il primo uomo a mettere piede sull'isola. Il posto gli piacque talmente tanto che una volta tornato in patria inviò dei coloni per abitare sia quell'isola che quelle circostanti.

Le due storie riportate da Strabone sono ricche di spunti per quanto riguarda la visione dei Persiani come signori delle acque. Paul Goukowsky ha suggerito in maniera, credo, convincente che il nome Erythras abbia un'origine molto lontana nel tempo essendo legata all'antroponimo sumerico Ziusudra (sum. ZI.U4 .SUD.RA), che ritroviamo trascritto in greco da Berosso nella forma Xisuthros. ${ }^{24}$ Nella mitologia mesopotamica

${ }^{20}$ Cfr. Briant 2002, 382-383.

21 Briant 2006, 9-75; Briant 2008, 155-218.

22 Il nome dell' isola che ospita la tomba di Erythras è controverso: Strabone riporta il toponimo Tyrine, Plinio il Vecchio VI. 153 la chiama Ogyris, mentre Arriano, Indike 37.2, che utilizza l'opera di Nearco, usa il toponimo Oaracta. L'isola di Oaracta corrisponderebbe all' odierna Qishm. Per una discussione delle questione e sulla figura di Mithropastes si veda Bucciantini (2015, 62-63), con relative bibliografia.

23 Burstein 1989, 43-45.

${ }^{24}$ Goukowsky 1974, 118, nota 39. Bosworth (2004, 66-70), ritiene invece che il nome Erythras sia il prodotto della fertile immaginazione di Mithropastes. 
Ziusudra è il re di Šuruppak (Tell Fara) che avvisato dal dio Enki/Ea dell'imminenza del Diluvio universale costruisce un'arca in cui salvare la sua famiglia e gli animali. Alla fine del cataclisma, sbarcherà nell'isola di Dilmun. A Nearco o ad Agatarchide sarebbe da attribuire la deformazione in Erythras, perché foneticamente vicino al più familiare termine Erythros. Vale la pena notare che i Persiani avevano assimilato questo mito mesopotamico e se ne erano appropriati: nel racconto riportato da Strabone ritroviamo in effetti la figura dell'eroe o del sovrano legata all'elemento dell'acqua e alla perizia tecnica che permette di avere la meglio sulle forze della natura con la costruzione di una imbarcazione.

Quali conclusioni possiamo trarre da questa breve rassegna sull'immagine dei Persiani in Strabone? Per quanto la dinastia achemenide e gli eventi a essa legati siano spesso riportati secondo il filtro dell'ideologia greca, la monarchia persiana non è presentata solo in luce negativa. Come ha fatto notare Eran Almagor, Strabone non adotta un atteggiamento univoco nei confronti dei barbari. ${ }^{25}$ Una delle interpretazioni possibili di questo fatto va forse ricercata nel retroterra culturale e nelle stesse origini familiari del geografo che erano l'esempio lampante (e da Strabone non rinnegato, ma al contrario promosso) di quel melting-pot etnico e culturale che caratterizzava l'Oriente ellenistico. Del resto, nel caso specifico di Strabone, non dobbiamo dimenticare che la sua famiglia era stata al servizio della dinastia del Ponto, che vantava con la Persia achemenide più che una semplice affinità ideologica, dal momento che affermava di discendere da Dario I. D'altra parte, Lebreton afferma che i Persiani sono regolarmente assimilati ai Parti di cui Strabone minimizza l'importanza. In questo modo, il geografo istituirebbe un'analogia tra le gesta di Alessandro contro i Persiani e di Augusto contro i Parti. ${ }^{26}$ Queste affermazioni, per quanto in parte condivisibili, vanno a mio modo di vedere sfumate e ricalibrate. Partiamo dalla presunta assimilazione dei Persiani con i Parti: in molti passi dei Geographikà le due civiltà sono senza dubbio accostate, ma il giudizio di valore dato da Strabone non è per questo identico. Prendiamo il passo sul mancato sfruttamento del commercio marittimo sul Caspio (XI.7.2). I Persiani e i Parti se ne sono disinteressati in quanto barbari, ma fra i due Strabone sostiene che i Parti "sono peggiori" dei Persiani nella gestione di questa situazione. ${ }^{27}$ Guardiamo poi il capitolo sulla Persia (XV.3.3), dove Strabone, dopo aver elencato le principali residenze reali achemenidi della regione, riporta quanto segue:

Queste (erano le residenze reali) ai tempi dell'impero persiano, mentre coloro che sono venuti dopo, com'è da aspettarsi, ne usarono altre meno sontuose. Difatti, la Perside è stata indebolita dai Macedoni e ancor più dai Parti, perché malgrado i Persiani siano ancora oggi governati da un loro re, tuttavia hanno perduto gran parte del loro potere e sono sottomessi al re dei Parti.

I Persiani achemenidi erano potenti, ma i Persiani dei tempi di Strabone (egli allude in questo caso ai dinasti di Perside noti dalle loro monete come frataraka) sono molto più deboli a causa non solo dei Macedoni, ma soprattutto dei Parti di cui sono divenuti vassalli. ${ }^{28}$

${ }^{25}$ Almagor 2005, 54-55.

${ }^{26}$ Lebreton 2011, 375.

${ }^{27}$ Si veda Coloru 2013, 35-39.

${ }^{28}$ Sui dinasti della Perside e sulla loro monetazione si vedano: Wiesehöfer 2005, 109-110, 119; Sarkhosh Curtis 2010, 379-394; Wiesehöfer 2012. 
In un passo successivo (XV.3.12) però si accenna ad una instabilità connaturata al dominio dei Parti:

Finché i Parti prosperano anche i loro sudditi prosperano, ma quando ci sono insurrezioni, cosa che capita spesso anche ai nostri giorni, gli esiti cambiano di volta in volta e non sono gli stessi per tutti: infatti i disordini portano un vantaggio ad alcuni, mentre per altri vanno contro le loro aspettative.

Questa debolezza è già stata messa in luce da Strabone al libro VI.4.2, dove l'autore sostiene che i Parti sono pronti a rimettere tutto il loro potere nelle mani dei Romani. ${ }^{29}$ Si ha dunque l'impressione che nell'ottica ellenica della storia degli scontri tra Asia ed Europa, i Persiani abbiano rappresentato un nemico più potente e per così dire onorevole rispetto a coloro che li hanno seguiti, in questo caso i Parti, la cui potenza è vista però come di qualità peggiore e più instabile al punto che a differenza di Alessandro, Augusto potrebbe impadronirsi del loro regno senza combattere. Anche per quanto riguarda il rapporto con lo spazio geografico, possiamo dire che i Persiani mantengono l'immagine di una grande potenza, barbara e superba - ma poteva essere diversamente? - capace di lasciare segni tangibili e duraturi nel paesaggio. Ai Parti in questo ambito è riservato il ruolo di epigoni, eredi di una tradizione di cui però non mostrano di essere all'altezza. Nei Geographikà assistiamo insomma al binomio potere e territorio. La potenza degli Achemenidi si manifesta sul terreno modificandone la toponimia, l'aspetto e piegandone le asperità col ricorso alla tecnica.

BIBLIOGRAFIA

Almagor, E. (2005), Who is a Barbarian? The Barbarians in the Ethnological and Cultural Taxonomies of Strabo, in: D. Dueck, H. Lindsay, S. Pothecary (eds.), Strabo's Cultural Geography: The Making of a Kolossourgia, New York: 42-55.

Bosworth, A.B. (2004) [rist. 1996], Alexander and the East: The Tragedy of Triumph, Oxford.

Briant, P. (2002), From Cyrus to Alexander: A History of the Persian Empire, Winona Lake.

Briant, P. (2006), Retour sur Alexandre et les katarraktes du Tigre : l'histoire d'un dossier (I), Studi Ellenistici 19: 9-75.

Briant, P. (2008), Retour sur Alexandre et les katarraktes du Tigre. II: (Suite et fin), Studi Ellenistici 20: $155-218$.

Bucciantini, V. (2015), Studio su Nearco di Creta. Dalla descrizione geografica alla narrazione storica, Alessandria.

Burstein, S.M. (1989), Agatharchides of Cnidus. On the Erythraean Sea, London.

Cohen G.M. (1995), The Hellenistic Settlements in Europe, the Islands, and Asia Minor, Berkeley-Los Angeles-Oxford.

Coloru, O. (2013), L'Hyrcanie arsacide à l'époque hellénistique. Un commentaire à Strabon, 11.7.1-5, Parthica 15: 31-42.

Cucinotta, E. (2011), Il proemio dei Persika di Cherilo di Samo. Una proposta di ricostruzione, Studi Classici e Orientali 57: 97-118.

${ }^{29}$ Drijvers 1998, 290. 
Dan, A. (2015), Grecs et Perses sur les Détroits : le démon enchaîné et la démesure du Grand Roi, Ancient West and East 14: 191-235.

Dąbrowa, E. (2015), L'histoire des Parthes dans la Géographie de Strabon, Studi Ellenistici 29: 285304.

Develin, R. (2003), Athenian Officials: 684-321 BC, Cambridge.

Drijvers, J.W. (1998), Strabo on Parthia and the Parthians, in: J. Wiesehöfer (hrsg.), Das Partherreich und seine Zeugnisse / The Arsacid Empire: Sources and Documentation, Beiträge des Internationalen Colloquiums, Eutin (27.-30. Juni 1996), Stuttgart: 279-293.

Goukowsky, P. (1974), Les juments du roi Erythras, REG 87: 111-137.

Lebreton S. (2011), Strabon d'Amasée, in: D. Lenfant (éd.), Les Perses vus par les Grecs. Lire les sources classiques sur l'Empire achéménide, Paris: 374-389.

Lenfant, D. (2004), Ctésias. La Perse. L'Inde. Autres fragments, Paris.

Lenfant, D. (éd.) (2011), Les Perses vus par les Grecs. Lire les sources classiques sur l'Empire achéménide, Paris.

Lerouge-Cohen, Ch. (2007), L'image des Parthes dans le monde gréco-romain de la fin du 1er siècle av. J.-C. Jusqu'à la fin du Haut-Empire romain, Stuttgart.

P'yankov, I.V. (1993), Cyropolis, EI 6: 514-515.

Potts, D.T. (2010), Achaemenid Interests in the Persian Gulf, in: J. Curtis, St. John Simpson (eds.), The World of Achaemenid Persia, London-New York: 523-534.

Rapin, Cl. (2013), On the Way to Roxane: The Route of Alexander the Great in Bactria and Sogdiana (328-327 BC), in: G. Lindström, S. Hansen, A. Wieczorek, M. Tellenbach (hrsg.), Zwichen Ost und West. Neue Forschungen zum antiken Zentralasien, Darmstadt: 43-82.

Rollinger, R. (2013), Alexander und die großen Ströme. Die Flußüberquerungen im Lichte altorientalischer Pioniertechniken, Wiesbaden.

Saprykin S.Ju. (2009), The Religion and Cults of the Pontic Kingdom: Political Aspects, in: J.M. Højte (ed.), Mithridates VI and the Pontic Kingdom, Aarhus: 249-275.

Sarkhosh Curtis, V. (2010), The Frataraka Coins of Persis: Bridging the Gap between Achaemenid and Sasanian Persia, in: J. Curtis, S. St John (eds.), The World of Achaemenid Persia. History, Art and Society in Iran and the Ancient Near East, London-New York: 379-394.

Tafażżolī, A. (1994), Derāz-dast, EI 7: 319-320.

Thollard, P. (1987), Barbarie et civilisation chez Strabon. Étude critique des livres III et IV de la Géographie, Paris.

Von Gall, H. (1990), The Figured Capitals at Taq-e Bostan and the Question of the So-Called Investiture in Parthian and Sasanian Art, Silk Road Art and Archaeology 1: 99-122.

Wiesehöfer, J. (2005), Ancient Persia: From 550 BC to 650 AD, Düsseldorf.

Wiesehöfer, J. (2012), Frataraka, EI, www.iranicaonline.org/articles/frataraka. 